\title{
Immunocytochemical and lesion studies support the hypothesis that the projection from the medial nucleus of the trapezoid body to the lateral superior olive is glycinergic
}

\author{
Sanford C. Bledsoe Jr. ${ }^{1}$, Colleen R. Snead ${ }^{1}$, Robert H. Helfert ${ }^{1}$, Vibhay Prasad ${ }^{1}$, \\ Robert J. Wenthold ${ }^{2}$ and Richard A. Altschuler ${ }^{1}$ \\ ${ }^{1}$ Kresge Hearing Research Institute, University of Michigan Medical School, Ann Arbor, MI 48109 (U.S.A.) \\ and $^{2}$ Laboratory of Neuro-otolaryngology, National Institutes of Health, Bethesda, MD 20205 (U.S.A.)
}

(Accepted 27 October 1989)

Key words: Glycine; Medial nucleus of the trapezoid body; Lateral superior olive; Kainic acid; Immunocytochemistry

\begin{abstract}
Pre- and postembedding immunocytochemical techniques were used to study the distribution of glycine immunoreactivity in the superior olivary complex of guinea pigs following kainic acid (KA) lesions of the medial nucleus of the trapezoid body (MNTB). Destruction of the MNTB by injecting 50-100 $\mathrm{nl}$ of $10 \mathrm{mM} \mathrm{KA}$ virtually abolished labeled neurons in the MNTB at the site of the lesion. This resulted in a marked decrease in the number of labeled fibers projecting to the ipsilateral lateral superior olive (LSO) and in the number of labeled fibers and presynaptic terminals in the neuropil of the LSO. Smaller volumes $(20 \mathrm{nl})$ of KA produced similar but more restricted changes that conformed to the topographic organization of the MNTB projection to the LSO. The results support the hypothesis that the MNTB to LSO pathway is glycinergic.
\end{abstract}

\section{INTRODUCTION}

The superior olivary complex (SOC) in the auditory brainstem contains 3 principal nuclei: the lateral superior olive (LSO), the medial superior olive (MSO) and the medial nucleus of the trapezoid body (MNTB). Each has a distinctive set of inputs and outputs connecting it with other parts of the auditory system (see refs. 2, 7, 15 for reviews). Within the SOC, principal cells of the MNTB exert an inhibitory influence on the activity of neurons in the homolateral $\mathrm{LSO}^{6,31,32,33}$, and there is increasing evidence that glycine is the neurotransmitter involved ${ }^{1,12}$, $19,23,25,27,29,36,38$. We tested this hypothesis using immunocytochemical techniques to study the effect of kainic acid (KA) lesions of the MNTB on the distribution of glycine immunoreactivity in the superior olivary complex. A preliminary report has been presented ${ }^{4}$.

\section{MATERIALS AND METHODS}

Experiments were performed on 12 adult guinea pigs (NIH strain) weighing $300-450 \mathrm{~g}$. They were anesthetized with ketamine hydrochloride $(30 \mathrm{mg} / \mathrm{kg}$ b.wt., i.m.) and xylazine ( $5 \mathrm{mg} / \mathrm{kg} \mathrm{b.wt.,} \mathrm{i.m.)}$ and held in a stereotaxic device with hollow ear bars for the delivery of sounds. Rectal temperature was monitored and maintained at 38 $\pm 0.5^{\circ} \mathrm{C}$ with a thermostatically controlled heating pad. To produce a unilateral lesion of the MNTB, an injection pipette, consisting of a glass micropipette (20-40 $\mu \mathrm{m}$ tip diameter) coupled to a $1 \mu \mathrm{l}$ Hamilton syringe, was lowered into the right MNTB from a dorsal approach through a small burr hole over the cerebellum. The site of the injection was determined using stereotaxic coordinates and near-field potentials recorded from the pipette in response to monaural and binaural clicks as previously described ${ }^{10}$. The pipette was positioned to pass through the medial edge of the superior paraolivary nucleus (SPN) to enter the medial aspect of the MNTB at a rostrocaudal location where all 3 principal nuclei of the olivary complex (MNTB, MSO and LSO) are present in transverse sections. To minimize mechanical damage to the tissue, $\mathrm{KA}(10 \mathrm{mM}$ in Ringer solution, pH 7.4) was slowly injected over $1 \mathrm{~min}$. Six animals were injected with a 50-100 nl volume of $\mathrm{KA}$ and 3 received a $20 \mathrm{nl}$ injection of the drug to reduce possible diffusion to adjacent olivary structures. Three control animals were injected with $100 \mathrm{nl}$ of Ringer solution alone.

After a 3-4 day survival period, the animals were anesthetized with chloral hydrate and perfused transcardially with $0.15 \mathrm{M}$ sodium cacodylate buffer ( $\mathrm{pH} 7.4$ ), followed by a mixed-aldehyde fixative in $0.1 \mathrm{M}$ sodium cacodylate buffer ( $\mathrm{pH} 7.4$ ). The fixative contained $4 \%$ paraformaldehyde and $0.2 \%$ glutaraldehyde for pre-embedding immunocytochemistry or $1 \%$ paraformaldehyde and $1.25 \%$ glutaraldehyde for postembedding immunocytochemistry. The brainstems were then removed, immersed in appropriate fixative for an additional $2-4 \mathrm{~h}$ and stored overnight at $4^{\circ} \mathrm{C}$ in $300-310 \mathrm{mOsm}$ phosphate-buffered saline (PBS), $\mathrm{pH}$ 7.4. Tissue from the 3 animals injected with $20 \mathrm{nl}$ of $\mathrm{KA}$ was processed by postembedding immunocytochemical techniques as described below. The brainstems of the remaining animals plus 2 normal (uninjected) guinea pigs were processed by conventional pre-embedding techniques. Transverse sections, cut on a vibratome at $60 \mu \mathrm{m}$, were pretreated in $0.015 \%$ hydrogen peroxide to lower endogenous peroxidase activity and incubated in PBS containing $10 \%$ normal goat serum

Correspondence: S. C. Bledsoe Jr., Kresge Hearing Research Institute, University of Michigan Medical School, 1301 E. Ann Street, Ann Arbor, MI 48109, U.S.A. 
(NGS) for $1 \mathrm{~h}$ to reduce non-specific binding. Free-floating sections were then incubated overnight at $4{ }^{\circ} \mathrm{C}$ with affinity-purified glycine antibody (described in detail previously ${ }^{36}$ ) diluted 1:800 in PBS containing $0.3 \%$ Triton X-100 detergent. Immunoperoxidase procedures using the avidin-biotin method ${ }^{21}$ and reagents from Vector Labs (Vectastain ABC Kit) were followed and peroxidase activity visualized for light microscopy using diaminobenzidine as the chromogen. In a few experiments, alternate sections were stained with thionin.

The brainstems of the 3 animals processed by postembedding immunocytochemistry, a method that improves punctate labeling of presynaptic terminals ${ }^{16}$, were vibratome-sectioned at $100-150 \mu \mathrm{m}$ in the transverse plane. The tissue was postfixed in $0.2 \%$ osmium tetroxide in $0.15 \mathrm{M}$ sodium cacodylate buffer ( $\mathrm{pH} \mathrm{7.4)}$ for $2 \mathrm{~h}$. The sections were then dehydrated through a graded ethanol series and propylene oxide, and embedded in EMbed 812-based resin (Electron Microscopy Supplies). The MNTB and LSO were dissected from the embedded tissue, sectioned at $1 \mu \mathrm{m}$ with an ultramicrotome and mounted onto glass microscope slides. To allow better penetration of antisera into the tissue, plastic was removed from the sections by an $11 \mathrm{~min}$ immersion in saturated sodium ethoxide (absolute ethanol saturated with sodium hydroxide) diluted 1:1 with absolute ethanol, followed by $\mathrm{dH}_{2} \mathrm{O}$ washes. To restore antigenicity, osmium was then bleached from the tissue by two 5-min immersions in $1 \%$ aqueous sodium meta-periodate, separated by a brief $\mathrm{dH}_{2} \mathrm{O}$ wash. Following additional $\mathrm{dH}_{2} \mathrm{O}$ rinses, the tissue was pretreated in blocking solution (5\% NGS in PBS) and incubated in a humidified chamber, at $4{ }^{\circ} \mathrm{C}$, for $48 \mathrm{~h}$ in glycine antisera diluted 1:400 in blocking solution. The procedures for visualizing the immunoreactivity were the same as in the pre-embedding method, except that the Vectastain reagents were used at twice the recommended concentration.

\section{RESULTS}

A distinctive pattern of glycine immunoreactive labeling was observed in neuronal cell bodies, fibers and terminals in the SOC of normal guinea pigs and in the SOC contralateral to the injection of KA (Fig. 1). The most intense labeling of cells occurred in the MNTB. On the basis of size and shape the majority of these neurons appeared to be the principal cells, which project to the ipsilateral LSO (Fig. 2A). The neuropil of the MNTB contained a meshwork of intensely immunoreactive fibers (Fig. 2A) and a few lightly labeled puncta, which are presumed to be presynaptic terminals. Light to moderately intense cell labeling was also observed in the SPN as well as in the lateral and ventral nuclei of the trapezoid body (LNTB and VNTB, respectively; Fig. 1). The neuropil of these nuclei contained a number of immunoreactive fibers and perisomatic puncta, which appeared to be most abundant in the SPN. In the MSO, the vast majority of the neurons showed no glycine labeling. However, the perikarya and proximal dendrites of unlabeled MSO neurons in the central cell band were apposed to immunoreactive puncta and coarse, intensely labeled fibers appeared to pass through and around the MSO enroute to the ipsilateral LSO. In the LSO, more than half of the neurons exhibited light glycine immunoreactivity (Fig. 3A). The size of the neurons ranged from 15 to $25 \mu \mathrm{m}$ and they were oval or fusiform in shape. The neuropil of the LSO contained an abundance of intensely labeled fibers and puncta (Figs. 3A and 4A). In addition, there was extensive perisomatic punctate labeling that was most readily apparent in the material processed by postembedding immunocytochemical techniques (Fig. 4A).

In animals injected with $50-100 \mathrm{nl}$ of KA, labeled neurons and fibers in the MNTB were virtually abolished at the site of the lesion (Figs. 1 and 2B). The extent of the lesions along the rostrocaudal axis was on the order of $300-500 \mu \mathrm{m}$, or approximately one-third to one-half of the total length of the MNTB. In addition to destroying the

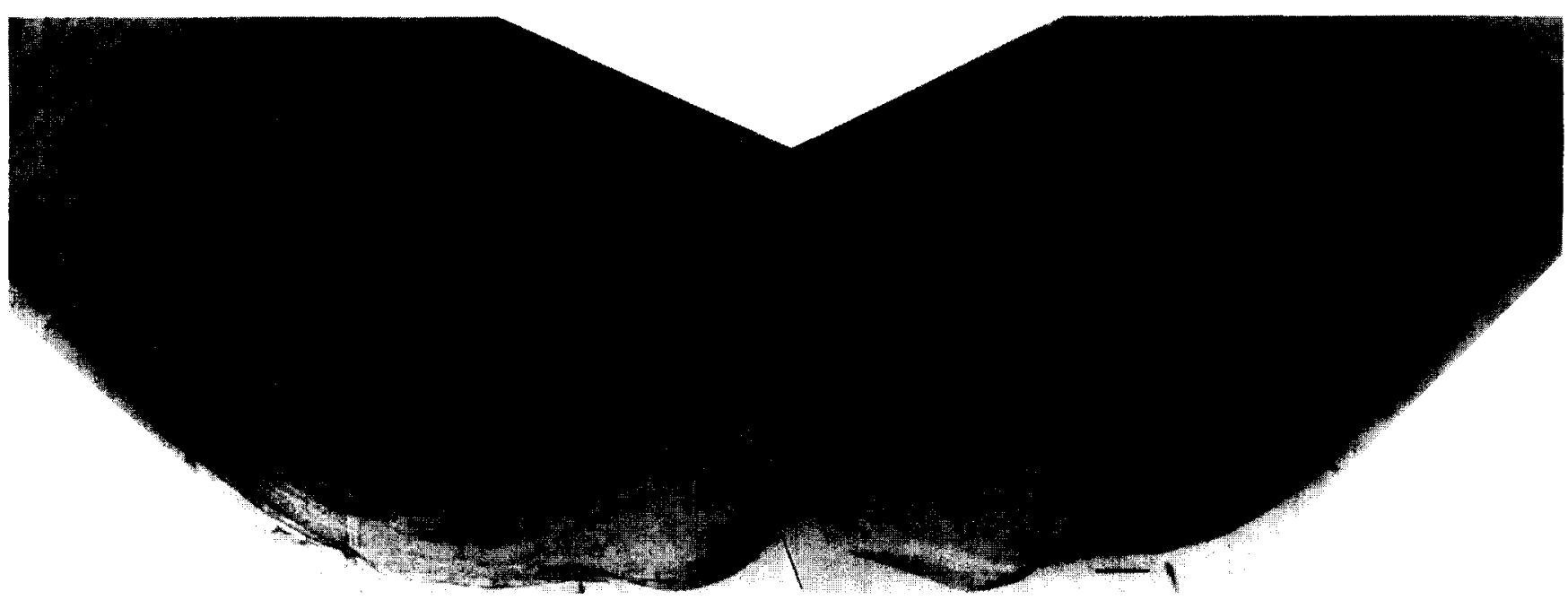

Fig 1. Transverse section through the guinea pig brainstem illustrating the effects of a KA lesion of the right MNTB on glycine immunoreactive labeling in the SOC. A normal distribution of immunolabeling is seen in the left SOC contralateral to the lesion. On the lesioned side there is a virtual absence of labeled cells in the lesioned MNTB and a marked decrease in the number of labeled fibers projecting throughout the neuropil of the SOC producing a slightly 'washed-out' appearance. 1, LSO; 2, SPN; 3, MNTB; 4, VNTB; 5, MSO; 6, LNTB. Volume of KA $=100 \mathrm{nl} ;$ bar $=250 \mu \mathrm{m}$. 

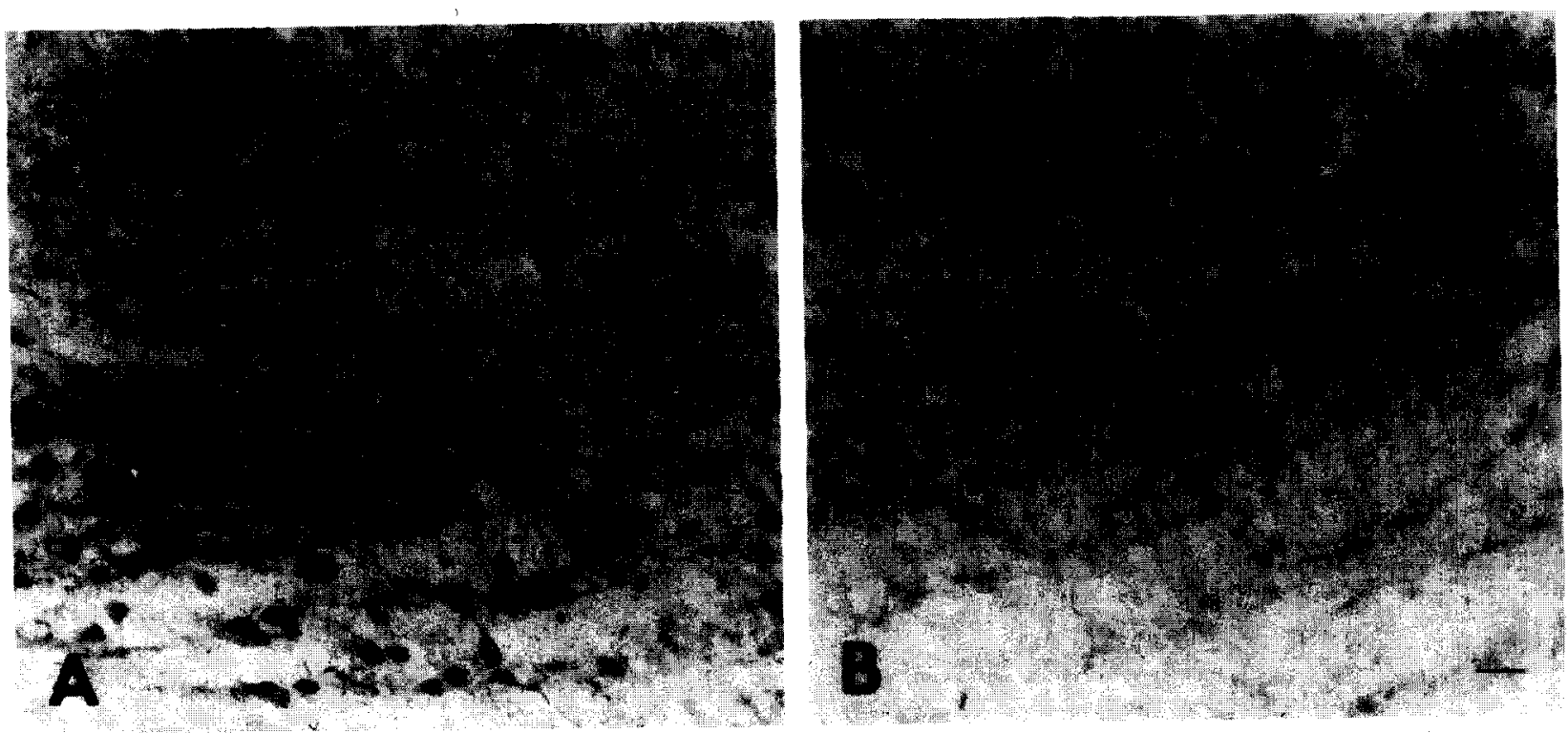

Fig. 2. Comparison of glycine immunoreactive labeling in the MNTB on the unlesioned (A) and lesioned (B) side of the brainstem. A: the large principal cells are intensely labeled as are many fibers in the neuropil. B: the labeling of neurons and fibers is virtually abolished. Volume of $\mathrm{KA}=50 \mathrm{nl}$; bar $=50 \mu \mathrm{m}$.

MNTB, KA invariably invaded the adjacent SPN and VNTB to produce partial or, in some instances, near-total neuronal destruction in these nuclei (Fig. 1). However,

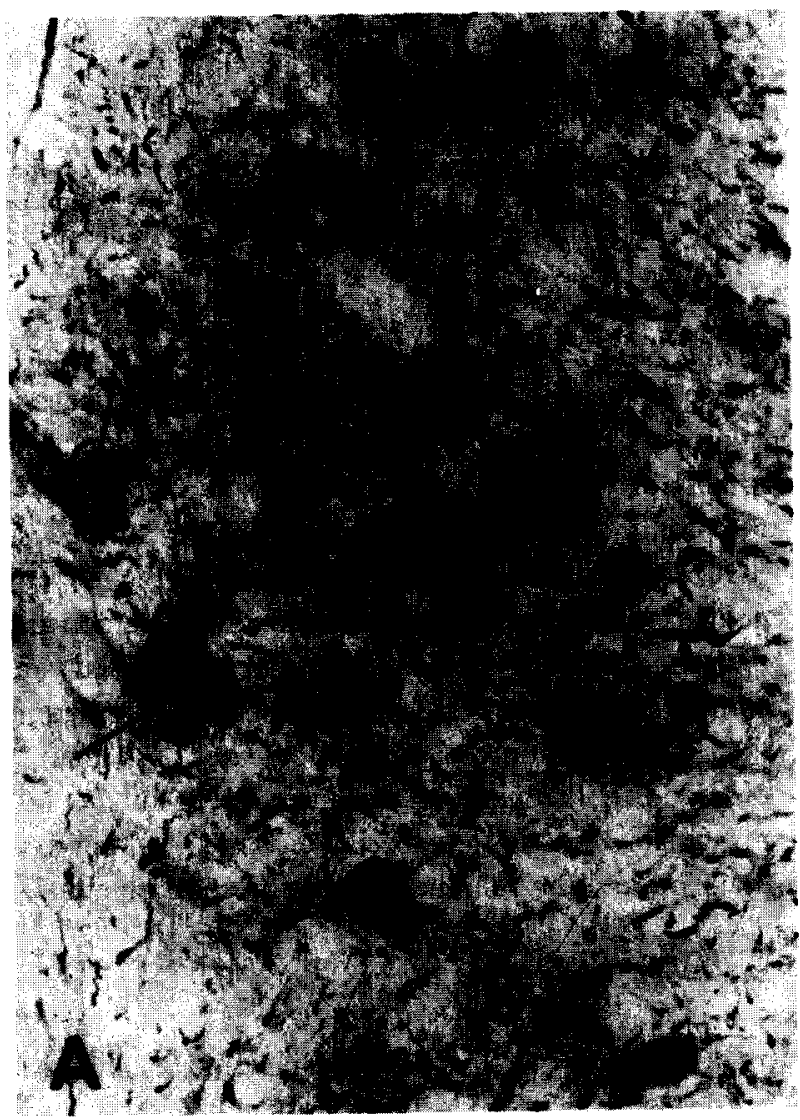

owing to the medial site of the injections (see Methods), there appeared to be little, if any, direct effect of KA on neurons in the nearby MSO and in the more lateral LSO.

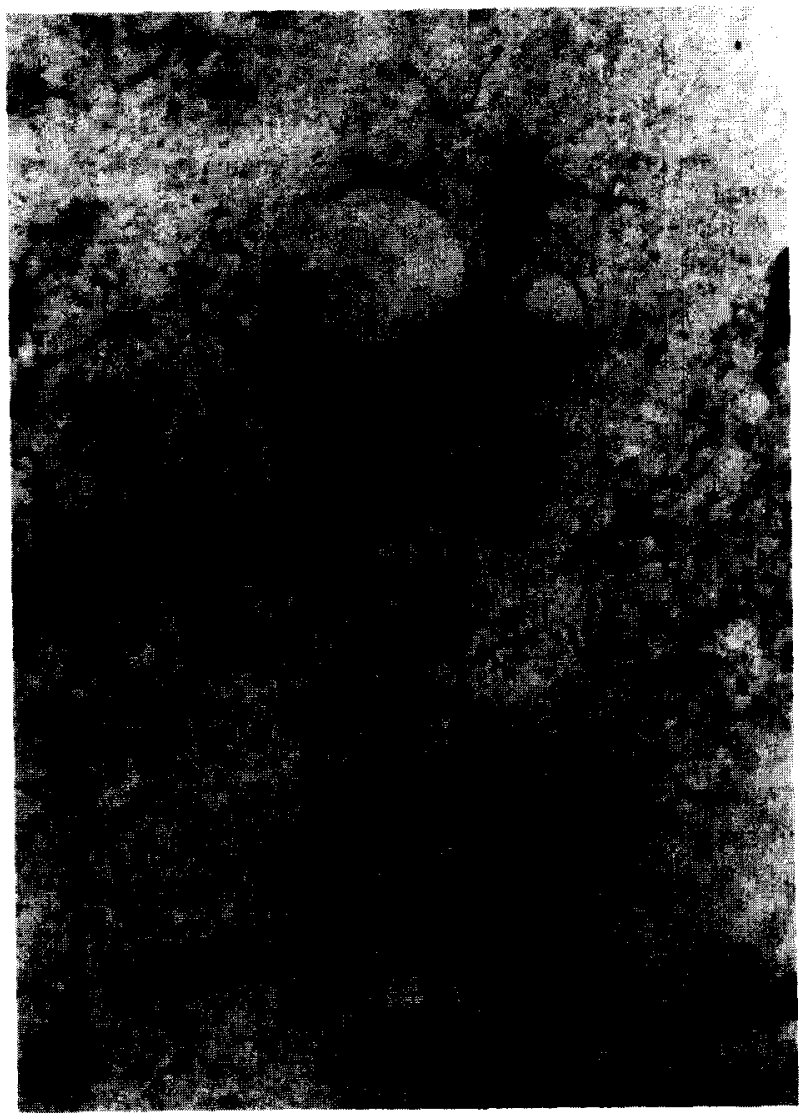

Fig. 3. Comparison of glycine immunoreactive labeling in the medial lobe of the LSO contralateral (A) and ipsilateral (B) to the injection of KA into the MNTB. A. The neuropil is rich with glycine immunolabeled fibers and puncta (see arrowheads for examples). Arrows indicate unlabeled and labeled cells with perisomatic punctate labeling $B$. There is a marked decrease in the number of labeled fibers and puncta in the neuropil as well as a decrease in perisomatic punctate labeling. Note the preservation of unlabeled and lightly immunopositive cell bodies (arrows) indicating little, if any, diffusion of KA from the MNTB. Volume of KA $=100 \mathrm{nl}$; bar $=20 \mu \mathrm{m}$. 

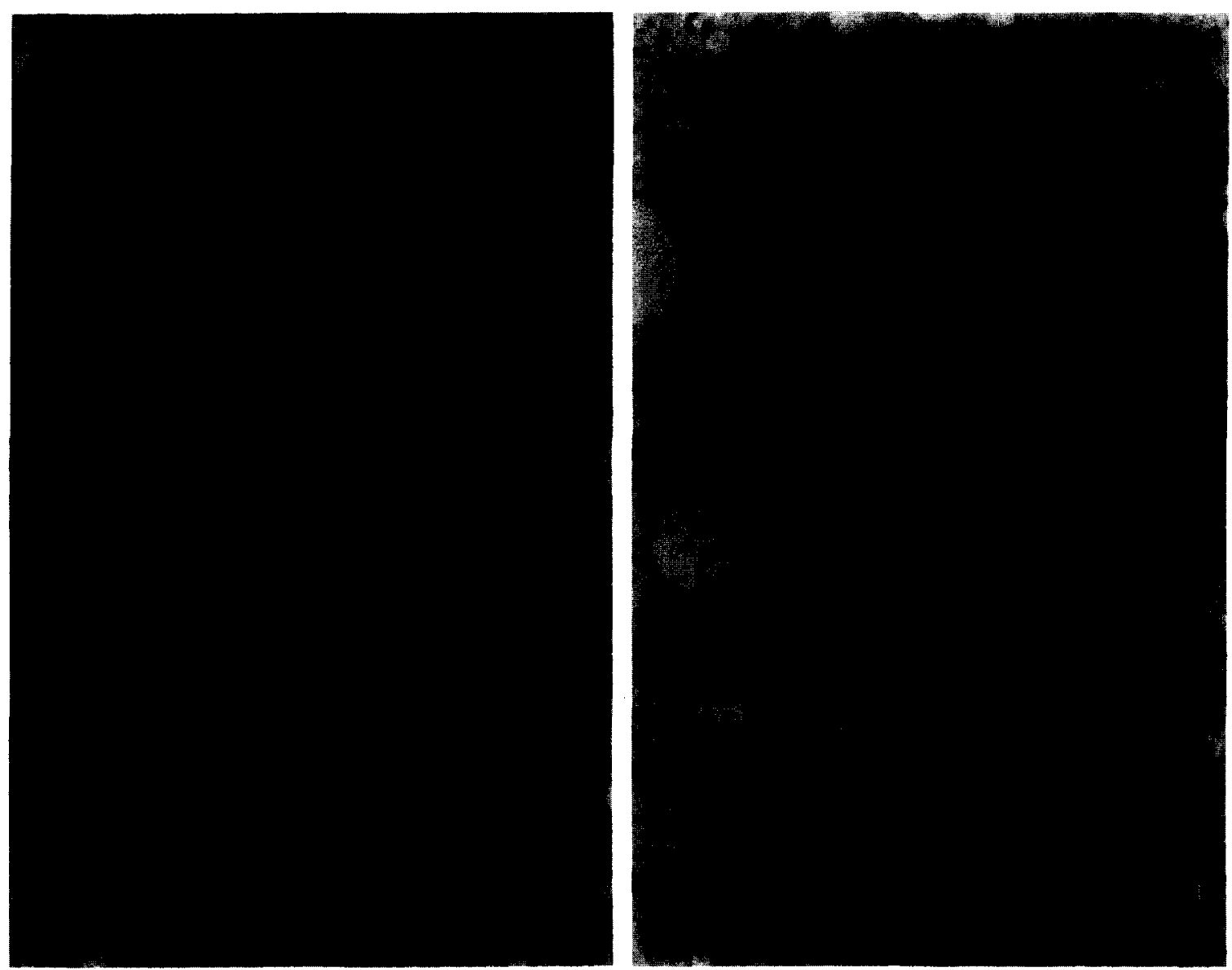

Fig. 4. Tissue processed by postembedding immunocytochemistry comparing glycine immunolabeling in the medial lobe of the LSO contralateral (A) and ipsilateral (B) to the injection of KA into the MNTB. A: the neuropil contains numerous labeled fibers and puncta. Arrows indicate cells with perisomatic punctate labeling. B: the number of labeled fibers and perisomatic puncta is greatly reduced. Volume of $\mathrm{KA}=20 \mathrm{nl}$; bar $=20 \mu \mathrm{m}$.

This was evidenced by the presence of neuronal cell bodies in Nissl stained material for the MSO, and both Nissl stained and immunolabeled sections for the LSO (Fig. 3B). Injections of $20 \mathrm{nl}$ of KA produced more discrete neuronal destruction, which was confined to the injection site in the medial aspect of the MNTB.

Concomitant to the destruction of the MNTB by $50-100$ $\mathrm{nl}$ of KA, there was a marked decrease in the number of labeled axons projecting to the ipsilateral LSO. There was also a widespread decrease in the number of labeled fibers and puncta in the neuropil of the LSO and an accompanying decrease, but not total abolishment, of perisomatic punctate labeling around LSO neurons (Figs. 3B and 4B). Smaller volumes $(20 \mathrm{nl})$ of $\mathrm{KA}$, which lesioned the medial aspect of the MNTB, produced a more restricted loss of labeled fibers and puncta that was confined to the medial lobe of the LSO. This is in keeping with the topographical arrangement of the projection of the MNTB to the $\mathrm{LSO}^{5,11,30}$, which provides the substrate for the tonotopic organization of these two nuclei $^{13,14,31}$.

With injections of $50-100 \mathrm{nl}$ of $\mathrm{KA}$ there was, in addition to the changes seen in the LSO, a general decrease in the number of labeled fibers and/or presynaptic terminals in the ipsilateral MSO, SPN, VNTB and LNTB. This accounts for the pale or slightly 'washed-out' appearance of the lesioned SOC compared to the contralateral SOC (Fig. 1). Control animals injected with Ringer solution alone demonstrated a pattern of labeling similar to normal (uninjected) animals with no discernable decrease in labeled fibers and puncta in the LSO, and only a slight decrease (less than 10\%) observed in the number of labeled cells in the MNTB; presumably, due to non-specific damage resulting from the injection and the presence of the injection pipette.

\section{DISCUSSION}

Results reveal that KA destruction of the MNTB produces a decrease in the number of glycine immunolabeled fibers and presynaptic terminals in the ipsilateral LSO. At the dilutions used in the present study, the anti-glycine antibody exhibits negligible cross reactivity with other neuroactive amino acids ${ }^{36}$. Thus, our findings 
support the hypothesis that the projection of the MNTB to the LSO is glycinergic. They also provide the first visual evidence linking glycine immunolabeled terminals in the LSO to neuronal cell bodies in the MNTB.

In the unlesioned SOC, intense immunolabeling was seen for cell bodies in the MNTB, and for fibers and terminals in the LSO. This pattern of labeling is compatible with recent immunocytochemical findings of Wenthold et al. ${ }^{36}$ and others ${ }^{1,19,27}$. It is well established from anatomical studies that neurons in the MNTB project to the ipsilateral LSO $^{11,26,30}$, thus completing the pathway to the LSO from the contralateral cochlear nucleus. Electron microscopic studies in cat $^{8,17}$ and rodent $^{18}$ have shown that a high percentage of the somatic and proximal dendritic surfaces of LSO principal neurons are contacted by synaptic terminals containing flattened synaptic vesicles. It has been presumed that this type of terminal corresponds to the perisomatic punctate glycine labeling seen in this and other immunocytochemical studies. This supposition receives support from a recent electron microscopic study by Helfert et al. ${ }^{20}$, which showed that glycine immunolabeled terminals contacting the somata and proximal dendrites of LSO principal cells contain flattened vesicles. In view of the inhibitory nature of the projection from MNTB to LSO, it is generally thought that the major source of these glycinergic terminals is the ipsilateral MNTB. This conclusion is directly substantiated by the results of the present study.

In addition to immunocytochemical data, other evidence suggests that glycine plays a neurotransmitter role in the superior olivary complex. Glycine concentrations are high in the SOC, especially in the $\mathrm{LSO}^{12}$. The numbers of glycine receptors, as measured autoradiographically with $\left[{ }^{3} \mathrm{H}\right]$ strychnine binding, have been shown to be exceptionally high in the LSO of cat $^{3}$ and rodent ${ }^{28,38}$. A similar observation has been reported in the guinea pig LSO by Wenthold et al. ${ }^{37}$ using a monoclonal antibody specific for the glycine postsynaptic receptor. In an autoradiographic study, Schwartz ${ }^{29}$ reported labeling of LSO synaptic terminals containing flattened vesicles after incubation with $\left[{ }^{3} \mathrm{H}\right]$ glycine. Moore and Caspary ${ }^{23}$, studying the iontophoretic application of glycine and strychnine in the chinchilla LSO, showed that glycine inhibited the excitatory response of LSO neurons to ipsilateral sound stimulation while strychnine blocked inhibition produced by both contralateral stimulation and the application of glycine. All these findings coupled with the present results provide compelling evidence that glycinergic neurons of the MNTB mediate contralateral inhibition in the LSO.

Although the principal cells of the homolateral MNTB provide the bulk of the contralateral input to the LSO, a direct projection from the cochlear nucleus to the contralateral LSO has been reported in $\operatorname{dog}^{13}$ and cat $^{11,34,35}$. Thus, one factor to consider in the interpretation of the present results is the ability of KA to selectively destroy neuronal cell bodies near the injection site while sparing axons passing through the injected area $^{9}$. Studying the acute effects of KA lesions of the MNTB on the scalp-recorded auditory brainstem response, Gardi and Bledsoe ${ }^{10}$ provided electrophysiological evidence that fibers passing through the lesioned MNTB are largely unaffected. Anatomical investigations by Masterton et al. ${ }^{22}$ and others ${ }^{11,30}$ have also shown the axon-sparing nature of KA lesions of the SOC after survival periods of several days. In the present study, no discernable degeneration of fibers and presynaptic terminals was observed in the contralateral SOC as evidenced by a normal pattern of glycine immunolabeling. This lack of effect in the contralateral SOC provides additional evidence that KA did not appreciably damage axons that pass through the injected MNTB enroute to the contralateral SOC.

The presence of neuronal perikarya in the MSO and LSO adjacent to the lesioned MNTB suggests that there was little lateral diffusion of KA to these olivary nuclei. Although cellular destruction invariably occurred in the juxtaposed SPN and VNTB following 50-100 nl injections of KA, neurons in these nuclei are not thought to project in substantial numbers to the LSO proper ${ }^{11,30}$. In view of these considerations, the decreased number of immunolabeled axons and terminals in the LSO appears most likely to be the result of neuronal loss confined to the vicinity of the injections in the ipsilateral MNTB.

Using Phaseolus vulgaris-leucoagglutinin (PHA-L), an anterograde axon tracer, we have recently demonstrated that, in addition to the well established projection of MNTB neurons to the ipsilateral LSO, guinea pig MNTB neurons also project ipsilaterally to the MSO, SPN, VNTB and LNTB ${ }^{5}$. These findings confirm and extend observations of others on the projection of MNTB principal cells to periolivary nuclei in $\mathrm{cat}^{24,30}$. In the present study immunoreactive fibers and axon terminals were observed, in varying numbers, in virtually all of the olivary nuclei. These most probably represent axons of passage and terminal fields of MNTB fibers and their collaterals. The extensive projection of MNTB neurons within the SOC may account for the widespread loss of immunolabeled fibers and axon terminals throughout the SOC following destruction of the MNTB with 50-100 $\mathrm{nl}$ of KA.

In conclusion, the results of this study provide additional evidence that the MNTB projection to the ipsilateral LSO is glycinergic. They also demonstrate that the use of axon-sparing excitotoxic lesions coupled with immunocytochemistry can yield valuable information about neurotransmitters utilized by specific populations of neurons. The technique, thus, serves as a useful 
adjunct to conventional axon-tracing techniques because transmitter candidates can be assigned to the synaptic relationships of neural circuits in auditory pathways.

\section{REFERENCES}

1 Aoki, E., Semba, R., Keino, H., Kato, K. and Kashiwamata, S., Glycine-like immunoreactivity in the rat auditory pathway, Brain Research, 442 (1988) 63-71.

2 Aitkin, L.M., Irvine, D.R.F. and Webster, W.R., Central neural mechanisms of hearing. In S.R. Geiger, I. Darian-Smith, J.M. Brookhart and V.B. Mountcastle (Eds.), Handbook of Physiology, The Nervous System, Vol. III, American Physiological Society, Bethesda, MD, 1984, pp. 675-737.

3 Baker, B.N., Glendenning, K.K. and Hodges, P., Acoustic chiasm: distribution of glycine and GABA receptors in the brainstem auditory nuclei of cat, Assoc. Res. Otolaryngol. Abstr., 9 (1986) 7.

4 Bledsoe, S.C., Jr., Altschuler, R.A., Wenthold, R.J. and Prasad, V., Immunocytochemical localization of glycine in the guinea pig superior olivary complex: lesion studies, Assoc. Res. Otolaryngol. Abstr., 10 (1987) 157.

5 Bledsoe, S.C., Jr., Pandya, P., Altschuler, R.A. and Helfert, R.H., Axonal projections of PHA-L labeled neurons in the medial nucleus of the trapezoid body, Soc. Neurosci. Abstr., 14 (1988) 491.

6 Boudreau, J.C. and Tsuchitani, C., Binaural interaction in the cat superior olive S segment, J. Neurophysiol., 31 (1968) 442-454.

7 Brugge, J.F. and Geisler, C.D., Auditory mechanisms of the lower brainstem, Annu. Rev. Neurosci., 1 (1978) 363-394.

8 Cant, N.B., The fine structure of the lateral superior olivary nucleus of the cat, J. Comp. Neurol., 227 (1984) 63-77.

9 Coyle, J.T., Molliver, M.E. and Kuhar, M.J., In situ injection of kainic acid: a new method for selectively lesioning neuronal cell bodies while sparing axons of passage, J. Comp. Neurol, 180 (1978) 301-324.

10 Gardi, J.N. and Bledsoe, S.C., The use of kainic acid for studying the origins of scalp-recorded auditory brainstem responses in the guinea pig, Neurosci. Lett., 26 (1981) 143-149.

11 Glendenning, K.K., Hutson, K.A., Nudo, R.J. and Masterton, R.B., Acoustic chiasm II: anatomical basis of binaurality in lateral superior olive of cat, J. Comp. Neurol., 232 (1985) 261-285.

12 Godfrey, D.A., Parli, J.A., Dunn, J.D. and Ross, C.D., Neurotransmitter microchemistry of the cochlear nucleus and superior olivary complex. In J. Syka and R.B. Masterton (Eds.), Auditory Pathway, Plenum, New York, 1988, pp. 107-121.

13 Goldberg, J.M. and Brown, P.B., Functional organization of the dog superior olivary complex: an anatomical and electrophysiological study, J. Neurophysiol., 31 (1969) 639-656.

14 Guinan, J.J., Jr., Norris, B.E. and Guinan, S.S., Single auditory units in the superior olivary complex. II. Locations of unit categories and tonotopic organization, Int. J. Neurosci., 4 (1972) 147-166.

15 Harrison, J.M., Functional properties of the auditory system of the brain stem. In R.B. Masterton (Ed.), Handbook of Behavioral Neurobiology, Plenum, New York, 1978, pp. 409-458.

16 Helfert, R.H. and Altschuler, R.A., unpublished observations.

17 Helfert, R.H. and Schwartz, I.R., Morphological evidence for the existence of multiple neuronal classes in the cat lateral superior olivary nucleus, J. Comp. Neurol., 244 (1986) 533-549.

18 Helfert, R.H. and Schwartz, I.R., Morphological features of five neuronal classes in the gerbil lateral superior olive, Am. J. Anat., 179 (1987) 55-69.

19 Helfert, R.H., Altschuler, R.A. and Wenthold, R.J., GABA and glycine immunoreactivity in the guinea pig superior olivary complex, Soc. Neurosci. Abstr., 13 (1987) 544.

20 Helfert, R.H., Bonneau, J.M., Wenthold, R.J. and Altschuler, R.A., Ultrastructural characterization of GABA and glycine
Acknowledgements. Supported by NIH Grants NS-05785, NS07106, NS-24369 and grants from The University of Michigan Medical Student Research Program and The Deafness Research Foundation.

immunoreactive synapses in the guinea pig superior olivary complex, Soc. Neurosci. Abstr., 14 (1988) 487.

21 Hsu, S.-M., Raine, L. and Fanger, H., Use of avidin-biotin -peroxidase complex (ABC) in immunoperoxidase techniques: a comparison between $\mathrm{ABC}$ and unlabeled antibody (PAP) procedures, J. Histochem. Cytochem., 29 (1981) 577-580.

22 Masterton, R.B., Glendenning, K.K. and Hutson, K.A., Preservation of trapezoid body fibers after biochemical ablation of superior olives with kainic acid, Brain Research, 173 (1979) 156-159.

23 Moore, M.J. and Caspary, D.M., Strychnine blocks binaural inhibition in lateral superior olivary neurons, J. Neurosci., 3 (1983) 237-242.

24 Morest, D.K., The collateral system of the medial nucleus of the trapezoid body of the cat, its neuronal architecture and relation to the olivocochlear bundle, Brain Research, 9 (1968) 288-311.

25 Peyret, D., Campistron, G., Geffard, M. and Aran, J.-M., Glycine immunoreactivity in the brainstem auditory and vestibular nuclei of the guinea pig, Acta Otolaryngol. (Stockh.), 104 (1987) 71-76.

26 Rasmussen, G.L., The olivary peduncle and other fiber connections of the superior olivary complex, J. Comp. Neurol., 84 (1946) 141-219.

27 Saint Marie, R.L., Ostapoff, E.-M., Morest, D.K. and Wenthold, R.J., A glycine-immunoreactive projection of the cat lateral superior olive: possible role in midbrain ear dominance, J. Comp. Neurol., 279 (1989) 382-396.

28 Sanes, D.H., Geary, W.A., Wooten, G.F. and Rubel, E.W., Quantitative distribution of the glycine receptor in the auditory brain stem of the gerbil, J. Neurosci., 7 (1987) 3793-3802.

29 Schwartz, I.R., Autoradiographic studies of amino acid labeling of neural elements in the auditory brainstem. In D.G. Drescher (Ed.), Auditory Biochemistry, C. Thomas, Springfield, 1985, pp. 258-277.

30 Spangler, K.M., Warr, W.B. and Henkel, C.K., The projections of principal cells of the medial nucleus of the trapezoid body in the cat, J. Comp. Neurol., 238 (1985) 249-262.

31 Tsuchitani, C., Functional organization of lateral cell groups of cat superior olivary complex, J. Neurophysiol., 40 (1977) 296-318.

32 Tsuchitani, C., The inhibition of cat superior olive unit excitatory responses to binaural tone bursts. I. The transient chopper response, J. Neurophysiol., 59 (1988) 164-183.

33 Tsuchitani, C., The inhibition of cat superior olive unit excitatory responses to binaural tone bursts. II. The sustained discharges, J. Neurophysiol., 59 (1988) 184-211.

34 Warr, B., Fiber degeneration following lesions in the multipolar and globular cell areas in the ventral cochlear nucleus of the cat, Brain Research, 40 (1972) 247-270.

35 Warr, W.B., Parallel ascending pathways from the cochlear nucleus: neuroanatomical evidence of functional specialization. In W.D. Neff (Ed.), Contributions to Sensory Physiology, Vol. 7, Academic Press, New York, 1982, pp. 1-38.

36 Wenthold, R.J., Altschuler, R.A., Huie, D., Parakkal, M.H. and Reeks, K.A., Glycine immunoreactivity localized in the cochlear nucleus and superior olivary complex, Neuroscience, 22 (1987) 897-912.

37 Wenthold, R.J., Betz, H., Reeks, K.A. Parakkal, M.H. and Altschuler, R.A., Localization of glycinergic synapses in the cochlear nucleus and superior olivary complex with monoclonal antibodies specific for the glycine receptor, Soc. Neurosci. Abstr., 11 (1985) 1048

38 Zarbin, J.M., Wamsley, J.K. and Kuhar, M.J., Glycine receptor: light microscopic autoradiographic localization with $\left[{ }^{3} \mathrm{H}\right]$ strychnine, J. Neurosci., 1 (1981) 532-547. 